\title{
Prandiales Insulin oder prandialer GLP-1-Rezeptoragonist?
}

\begin{abstract}
Die zusätzliche Gabe eines Basalinsulins zu oralen Antidiabetika ist der bewährte Einstieg in die Insulintherapie mit dem Ziel, den Nüchternblutzucker zu normalisieren. Wenn dann eine Therapieintensivierung nötig wird, so empfiehlt sich besonders bei stark übergewichtigen Patienten ein prandialer GLP-1-Agonist als Alternative zum prandialen Insulin.
\end{abstract}

Wenn bei Typ-2-Diabetikern mit oralen Antidiabetika (OAD) die Zielwerte nicht erreicht werden, besteht die Indikation für ein Basalinsulin wie Insulinglargin (Lantus $\left.{ }^{\circledR}\right)$ zusätzlich. „Durch eine frühzeitige Insulintherapie wird die basale Hyperglykämie und damit der Nüchternblutzucker reduziert", sagte Prof. Gerhard H. Scholz aus Leipzig. Dadurch werde auch die hepatische Glukoseproduktion der Leber gehemmt mit konsekutiver Verbesserung der Betazellfunktion. Doch können auch damit die Zielwerte nicht erreicht werden, muss die Therapie weiter intensiviert werden, um die postprandiale Hyperglykämie zu reduzieren. Nun könne ein prandiales Insulin oder ein prandialer GLP-1-Agonist helfen, so Scholz. Entscheide man sich fürs Insulin, genüge meist schon ein kurz wirksames Analogon wie Insulinglulisin (Apidra ${ }^{\circledR}$ ) zur Hauptmahlzeit. Durch die zusätzliche Gabe von Glargin zu OAD konnte in Studien nach 16 Wochen der $\mathrm{HbA}_{1 \mathrm{c}}$ um $1,1 \%$ gesenkt werden, und durch die $\mathrm{Zu}$ gabe von Glulisin wird er um weitere $0,8 \%$ gesenkt, so Scholz.

\section{Additive und synergistische Wirkung} Für stark übergewichtige Patienten etwa dürfte aber ein GLP-1-Agonist wie Lixisenatid (Lyxumia ${ }^{\circ}$ ) sinnvoller sein, zumal

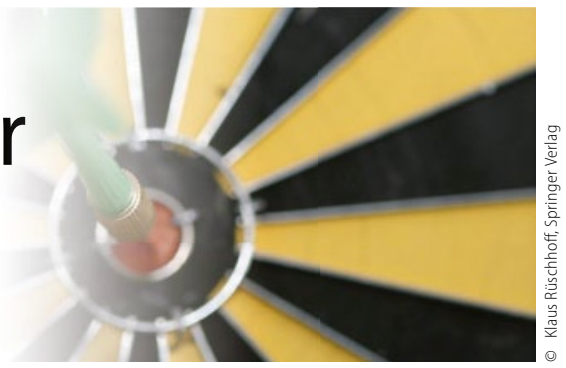

er auch nur einmal täglich prandial gegeben werden muss. Basalinsulin und GLP1-Agonisten entfalten nicht nur synergistische, sondern auch additive Wirkungen. So wird durch den GLP-1-Agonisten nicht nur die Insulin-Biosynthese und die Betazellfunktion und somit die Insulinsekretion gesteigert und die Glukagonsekretion vermindert. Die Substanz senkt auch den Appetit und verzögert die Magenentleerung, was zu einer Gewichtsreduktion führt. „Durch Lixisenatid wird nicht nur der postprandiale Blutzucker und der $\mathrm{HbA}_{1 \mathrm{c}}$ gesenkt, sondern auch die Blutzuckerschwankungen werden geglättet", so Scholz. Das sei im Hinblick auf vaskuläre Folgen vorteilhaft.

Dr. med. Peter Stiefelhagen

Quelle: Satellitensymposium beim Diabeteskongress am 8.5.2013 in Leipzig, Veranstalter: Sanofi
Alle Accu-Chek ${ }^{\circledR}$-Blutzuckermesssysteme erfüllen Anforderungen der neuen ISO-Norm zur Messgenauigkeit

Um eine europaweite Marktzulassung zu erhalten, müssen Blutzuckermessgeräte eine CE-Kennzeichnung haben. Diese bestätigt aber nur die grundsätzliche Funktionstüchtigkeit der Geräte. Daher ist es nötig, vor allem die Messqualität anhand standardisierter Vorgaben wie die der ISO-Norm zu ermitteln. Nur so können zuverlässige Testergebnisse als Grundlage für die Berechnung der Insulindosis dienen.

Am 14. Mai 2013 ist die überarbeitete und verschärfte Version der DIN ISO-Norm 15197 zur Qualitätssicherung von Blutzuckermessgeräten und Teststreifen publiziert worden, die die alte Fassung von 2003 ersetzt. 2012 wurde die Messgenauigkeit von 34 Geräten und den passenden Teststreifen in zwei Studien anhand der alten und neuen Fassung der ISO-Norm geprüft. 80\% der Geräte erfüllten die Vorgaben der alten ISO-Norm, wohingegen beinahe jedes zweite den Standard der neuen nicht einhalten konnte. Roche Diagnostics erfüllt mit allen Accu-Chek ${ }^{\circledR}$ Messsystemen sowohl die Anforderungen der alten als auch die verschärften Genauigkeitskriterien der neuen ISO-Norm 15197:2013. Neben den höheren Ansprüchen an die Messgenauigkeit regelt die neue Norm auch andere Parameter wie die Handhabung der Blutzuckermessgeräte.
Mutmacher für Kinder mit Diabetes: Die Fine Stars 2013

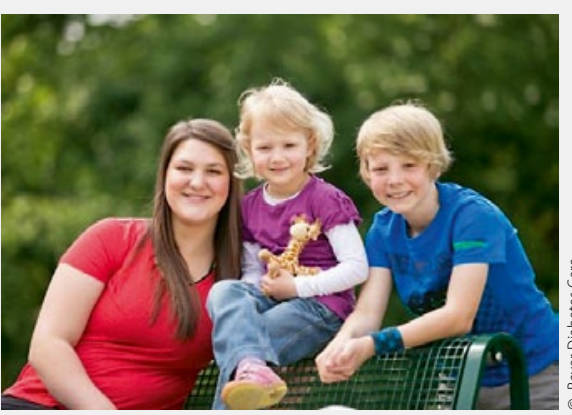

Bereits das dritte Mal kürte Bayer seine Fine Stars: drei starke Kids mit Diabetes, die anderen betroffenen Kindern und Jugendlichen mit ihrer Geschichte Mut machen wollen. Laila (5) aus München, Timon (10) aus Frankenberg und Johanna (16) aus Hildesheim haben ihren individuellen Weg gefunden, mit Diabetes umzugehen, sich von der Krankheit nicht unterkriegen zu lassen, und wollen dies anderen Kindern zeigen. Als Botschafter für Gleichaltrige ging es für die Kids im Mai zum Fine Stars-Fotoshooting nach Köln. Entstanden sind fröhliche Bilder - überzeugen Sie sich selbst. Bilder und weitere Infos unter http://www.diabetes.bayer.de/kids/fine-star/fine-stars eb

Quelle: Pressemitteilung von Bayer Diabetes Care vom 19.6.2013 